\title{
Dispersive Calculation of the Massless Multi-loop Sunrise Diagram
}

\author{
ANDREAS ASTE \\ Department of Physics and Astronomy, Theory Division, University of Basel, \\ Klingelbergstrasse 82, 4056 Basel, Switzerland.e-mail: andreas.aste@unibas.ch
}

Received: 10 March 2006; revised version: 7 May 2006

Published online: 15 June 2006

\begin{abstract}
The massless sunrise diagram with an arbitrary number of loops is calculated in a simple but formal manner. The result is then verified by rigorous mathematical treatment. Pitfalls in the calculation with distributions are highlighted and explained. The result displays the high energy behaviour of the massive sunrise diagrams, whose calculation is involved already for the two-loop case.
\end{abstract}

Mathematics Subject Classification (2000). 81T05, 81T15, 81T18.

Keywords. regularization, causality, perturbative calculations.

\section{Introduction}

The Feynman propagator of a free massless scalar quantum field $\Phi(x)$ fulfilling the wave equation

$$
\square \Phi(x)=\partial_{\mu} \partial^{\mu} \Phi(x)=0
$$

is given in configuration space by the vacuum expectation value

$$
\begin{aligned}
\Delta_{F}(x) & =-i\langle 0|T(\Phi(x) \Phi(0))| 0\rangle \\
& =\int \frac{\mathrm{d}^{4} k}{(2 \pi)^{4}} \frac{\mathrm{e}^{-i k x}}{k^{2}+i 0} \\
& =\frac{i}{4 \pi^{2}} \frac{1}{x^{2}-i 0}=\frac{i}{4 \pi^{2}} P \frac{1}{x^{2}}-\frac{1}{4 \pi} \delta\left(x^{2}\right),
\end{aligned}
$$

where $T$ is the time-ordering operator, $P$ denotes principal value regularization and $\delta$ is the one-dimensional Dirac distribution depending on $x^{2}=x_{\mu} x^{\mu}=$ $\left(x^{0}\right)^{2}-\left(x^{1}\right)^{2}-\left(x^{2}\right)^{2}-\left(x^{3}\right)^{2}=x_{0}^{2}-\vec{x}^{2}$. Formally, one could write for the massless $(n-1)$-loop sunrise diagram 


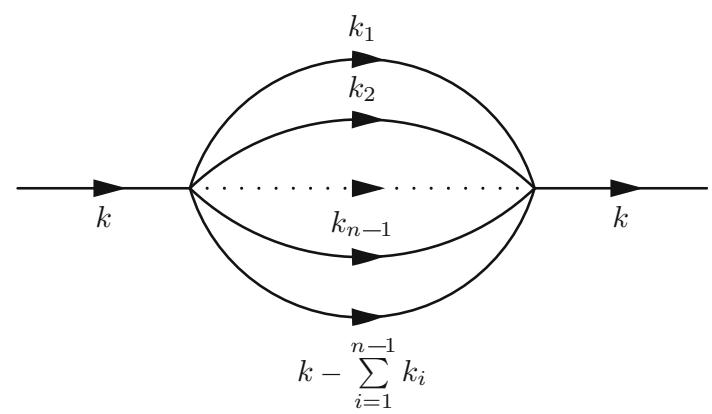

Figure 1. The $(n-1)$-loop sunrise diagram.

$$
\begin{aligned}
\int \mathrm{d}^{4} x\left[\Delta_{F}(x)\right]^{n} \mathrm{e}^{i k x}= & \Gamma_{n}(k) \\
= & \int \frac{\mathrm{d}^{4} k_{1}}{(2 \pi)^{4}} \ldots \frac{\mathrm{d}^{4} k_{n-1}}{(2 \pi)^{4}} \frac{1}{k_{1}^{2}+i 0} \frac{1}{k_{2}^{2}+i 0} \ldots \\
& \times \frac{1}{\left(k-k_{1}-k_{2}-\cdots-k_{n-1}\right)^{2}+i 0} .
\end{aligned}
$$

This expression above is both ultraviolet and infrared divergent for $n \geqslant 2$, consequently the corresponding expressions in Eq. (3) are all ill-defined. Therefore, $\left[\Delta_{F}(x)\right]^{n}$ has to be regularized in order to obtain a mathematically well-defined tempered distribution in the dual Schwartz space $\mathcal{S}^{\prime}\left(\mathbb{R}^{4}\right)$. The regularization can be performed by one of the many well-known methods [1-6], which all lead to the same result. For $n=2$ one obtains

$$
\begin{aligned}
\Gamma_{2}^{\mathcal{R}}(k) & =\mathcal{R}\left[\int \frac{\mathrm{d}^{4} k_{1}}{(2 \pi)^{4}} \frac{1}{k_{1}^{2}+i 0} \frac{1}{\left(k-k_{1}\right)^{2}+i 0}\right] \\
& =-\frac{i}{4(2 \pi)^{2}} \log \left(-\frac{k^{2}+i 0}{\mu_{\mathcal{R}}^{2}}\right)=-\frac{i}{4(2 \pi)^{2}} \log \left|k^{2} / \mu_{\mathcal{R}}^{2}\right|-\frac{1}{16 \pi} \Theta\left(k^{2}\right),
\end{aligned}
$$

where $\mathcal{R}$ denotes the regularization procedure with renormalization scale $\mu_{\mathcal{R}}$ and $\Theta$ is the Heaviside distribution. Obviously, the regularized expression for the oneloop diagram $\Gamma_{2}^{\mathcal{R}}(k)$ is defined up to a constant im momentum space or up to a local distribution in configuration space $\sim \delta^{(4)}(x)$. The scaling symmetry of the Feynman propagator $\Delta_{F}(\lambda x)=\lambda^{-2} \Delta_{F}(x), \lambda \in \mathbb{R}$, is spontaneously broken by the regularization procedure, such that a corresponding scaling law $\Gamma_{2}^{\mathcal{R}}(\lambda k)=\Gamma_{2}^{\mathcal{R}}(k)$ does not hold as one might expect naively from the formal definition of $\Gamma_{2}(k)$ in Eq. (3). 


\section{Formal Approach}

In order to obtain the analytic result for an arbitrary multi-loop diagram, a purely formal calculation is presented here first. One easily derives formally for $n \neq 1$

$$
\square \frac{1}{\left(x^{2}-i 0\right)^{n}}=\frac{4 n(n-1)}{\left(x^{2}-i 0\right)^{n+1}},
$$

and therefore

$$
\left[\Delta_{F}(x)\right]^{n}=-\frac{i^{n}}{4^{n-2}(2 \pi)^{2(n-2)}(n-1) !(n-2) !} \square^{n-2}\left[\Delta_{F}(x)\right]^{2} .
$$

Since the d'Alembert operator $\square$ goes over into $-k^{2}$ in momentum space, one obtains

$$
\Gamma_{n}^{\mathcal{R}}(k)=-\frac{(-i)^{n}\left(k^{2}\right)^{n-2}}{4^{n-2}(2 \pi)^{2(n-2)}(n-1) !(n-2) !} \Gamma_{2}^{\mathcal{R}}(k)+\sum_{i=0}^{n-2} c_{i}^{\mathcal{R}}\left(k^{2}\right)^{i}, \quad n \geqslant 2,
$$

if the assumption is made that the formal result eq. (6) is also valid for the regularized expressions. The additional terms involving the Fourier transform of (covariant derivatives of) the $\delta$-distribution in configuration space reflect the fact that the regularized Lorentz invariant product of Feynman propagators is only defined up to local terms given by derivatives of the $\delta$-distribution, whose order can be restricted by the requirement that the scaling behaviour of (7) corresponds to the artificial degree of divergence $\omega=2(n-2)$ of the $(n-1)$-loop sunrise diagram. Further restrictions follow from physical symmetry requirements, depending on the underlying theory in which the diagram plays a role (like in perturbative quantum gravity). As an example, for the 2-loop diagram $(n=3)$ one has the general result

$$
\Gamma_{3}^{\mathcal{R}}(k)=-\frac{k^{2}}{32(2 \pi)^{4}} \log \left(-\frac{k^{2}+i 0}{\mu_{\mathcal{R}}^{2}}\right)+c_{0}^{\mathcal{R}} .
$$

The simple result given by Eq. (7) is indeed correct and similar formal calculations as presented above can be found in many textbooks (see, e.g., [7]). The massive two-loop case of the sunrise diagram which is already involved from the computational point of view has been studied in detail in numerous papers (see e.g. [818] and references therein), and to a certain extent also for the massive multi-loop case [19-22].

\section{Properties of Tempered Distributions}

A rigorous derivation of (7) is presented below. For the forthcoming discussion, it is advantageous to review some of the useful properties of basic tempered distributions appearing in quantum field theory. A scalar neutral field $\Phi$ can be decomposed into a negative and positive frequency part according to 


$$
\Phi(x)=\Phi^{-}(x)+\Phi^{+}(x)=\int \frac{\mathrm{d}^{3} k}{\sqrt{(2 \pi)^{3} 2|\vec{k}|}}\left[a(\vec{k}) \mathrm{e}^{-i k x}+a^{\dagger}(\vec{k}) \mathrm{e}^{i k x}\right] .
$$

The commutation relations for such a field are given by the positive and negative frequency Jordan-Pauli distributions

$$
\Delta^{ \pm}(x)=-i\left[\Phi^{\mp}(x), \Phi^{ \pm}(0)\right]=-i\left\langle 0\left|\left[\Phi^{\mp}(x), \Phi^{ \pm}(0)\right]\right| 0\right\rangle,
$$

which have the Fourier transforms

$$
\hat{\Delta}^{ \pm}(k)=\int \mathrm{d}^{4} x \Delta^{ \pm}(x) \mathrm{e}^{i k x}=\mp(2 \pi i) \Theta\left( \pm k^{0}\right) \delta\left(k^{2}\right) .
$$

The fact that the commutator

$$
[\Phi(x), \Phi(0)]=i \Delta^{+}(x)+i \Delta^{-}(x)=: i \Delta(x)
$$

vanishes for spacelike arguments $x^{2}<0$ due to the requirement of microcausality, leads to the important property that the Jordan-Pauli distribution $\Delta$ has causal support, i.e. it vanishes outside the closed forward and backward lightcone such that

$$
\operatorname{supp} \Delta(x) \subseteq \bar{V}^{-} \cup \bar{V}^{+}, \quad \bar{V}^{ \pm}=\left\{x \mid x^{2} \geqslant 0, \pm x^{0} \geqslant 0\right\}
$$

in the sense of distributions. A further crucial observation is the fact that one can introduce the retarded propagator $\Delta^{\text {ret }}(x)$ which coincides with $\Delta(x)$ on $\bar{V}^{+}-\{0\}$, i.e. $\left\langle\Delta^{\text {ret }}, \varphi\right\rangle=\langle\Delta, \varphi\rangle$ holds for all test functions in the Schwartz space $\varphi \in \mathcal{S}\left(\mathbb{R}^{4}\right)$ with support $\operatorname{supp} \varphi \subset \mathbb{R}^{4}-\bar{V}^{-}$. One might be tempted to write down in configuration space

$$
\Delta^{\mathrm{ret}}(x)=\Theta\left(x^{0}\right) \Delta(x),
$$

and to transform this expression into a convolution in momentum space

$$
\hat{\Delta}^{\text {ret }}(k)=\int \frac{d^{4} p}{(2 \pi)^{4}} \hat{\Delta}(p) \hat{\Theta}(k-p), \quad \hat{\Delta}(p)=-(2 \pi i) \operatorname{sgn}\left(p^{0}\right) \delta\left(p^{2}\right) .
$$

The Heaviside distribution $\Theta\left(x^{0}\right)$ could be replaced by $\Theta(v x)$ with an arbitrary vector $v \in V^{+}=\bar{V}^{+}-\partial \bar{V}^{+}$inside the open forward lightcone. The Fourier transform of the Heaviside distribution $\Theta\left(x^{0}\right)$ can be calculated easily

$$
\hat{\Theta}(k)=\lim _{\epsilon \rightarrow 0} \int \mathrm{d}^{4} x \Theta\left(x^{0}\right) \mathrm{e}^{-\epsilon x^{0}} \mathrm{e}^{i k_{0} x^{0}-i \vec{k} \vec{x}}=\frac{(2 \pi)^{3} i}{k^{0}+i 0} \delta^{(3)}(\vec{k}) .
$$

For the special case where $k$ is in the forward lightcone $V^{+}$, one can go to a Lorentz frame where $k=\left(k^{0}, \overrightarrow{0}\right)$ such that Eq. (15) becomes

$$
\hat{\Delta}^{\mathrm{ret}}\left(k^{0}, \overrightarrow{0}\right)=\frac{i}{2 \pi} \int \mathrm{d} p^{0} \frac{\hat{\Delta}\left(p^{0}\right)}{k^{0}-p^{0}+i 0}=\frac{i}{2 \pi} \int \mathrm{d} t \frac{\hat{\Delta}\left(t k^{0}\right)}{1-t+i 0} .
$$


Hence, for arbitrary $k \in V^{+}, \hat{\Delta}^{\text {ret }}$ would be given by the dispersion relation

$$
\hat{\Delta}^{\mathrm{ret}}(k)=\frac{i}{2 \pi} \int \mathrm{d} t \frac{\hat{\Delta}(t k)}{1-t+i 0} .
$$

However, the integral in eq. (18) is undefined. One can circumvent this problem, e.g., by introducing a mass for the field $\Phi$ as a regulator for the $\Delta$-distribution. For the massive Jordan-Pauli distribution $\Delta_{m}(x)$ one gets due to the $\delta$-distribution in

$$
\hat{\Delta}_{m}(k)=-(2 \pi i) \operatorname{sgn}\left(k^{0}\right) \delta\left(k^{2}-m^{2}\right)
$$

the expression $\left(k \in V^{+}\right)$

$$
\begin{aligned}
\hat{\Delta}_{m}^{\mathrm{ret}}(k) & =\int \mathrm{d} t \frac{\operatorname{sgn}\left(t k^{0}\right) \delta\left(t^{2} k^{2}-m^{2}\right)}{1-t+i 0} \\
& =\int \mathrm{d} t \frac{\left[\delta\left(t-\frac{m}{\sqrt{k^{2}}}\right)-\delta\left(t+\frac{m}{\sqrt{k^{2}}}\right)\right]}{2 \sqrt{k^{2}} m(1-t+i 0)}=\frac{1}{k^{2}-m^{2}} \quad\left(k^{2} \neq m^{2}\right) .
\end{aligned}
$$

As a special case of the edge of the wedge theorem [23] it is known that the Fourier transform of the retarded distribution $\hat{\Delta}^{\text {ret }}(k)$ is the boundary value of an analytic function $r(z)$, regular in $T^{+}:=\mathbb{R}^{4}+i V^{+}$. This way one obtains from $r(z)=$ $1 /\left(z^{2}-m^{2}\right), z \in T^{+}$, and $m \rightarrow 0$

$$
\hat{\Delta}^{\mathrm{ret}}(k)=\frac{1}{k^{2}+i k^{0} 0} .
$$

The analytic expression for the Feynman propagator is then recovered, which coincides with

$$
\hat{\Delta}^{\mathrm{ret}}(k)=\hat{\Delta}_{F}(k)+\Delta^{-}(k)=\frac{1}{k^{2}+i k^{0} 0}
$$

for $k \in V^{+}$. The singular part of the Feynman propagator is given by

$$
\operatorname{Im}\left(\hat{\Delta}_{F}(k)\right)=-\pi \delta\left(k^{2}\right)
$$

and can be deduced directly from the causal Jordan-Pauli distribution $\hat{\Delta}$ in an obvious way.

\section{Analysis of Causal Properties}

The causal properties of the Jordan-Pauli distribution and the retarded propagator can be observed in an analogous way in the case of the distributions describing a multi-loop sunrise diagram. The normally ordered product of $n$ free field operators : $\Phi(x)^{n}$ : is an operator valued distribution. Therefore, the same is true for the tensor product : $\Phi(x)^{n}:: \Phi(y)^{n}:$ [24]. The vacuum expectation value of $\left\langle 0\left|: \Phi(x)^{n}:: \Phi(0)^{n}:\right| 0\right\rangle$ can be extracted by Wick's theorem and leads to $i^{n} n ! \Delta^{+}(x)^{n}$ 
(the combinatorial factor $n$ ! will be disregarded in the following), which is indeed a well-defined tempered distribution. Calculating the retarded part of the causal distribution $\Delta_{n}(x)$ which we define for $n \geqslant 2$ by

$$
\Delta_{n}(x):=i^{n}\left(\Delta^{+}(x)^{n}-(-1)^{n}\left(\Delta^{-}(x)^{n}\right)=\frac{1}{n !}\left\langle 0\left|\left[: \Phi(x)^{n}:,: \Phi(0)^{n}:\right]\right| 0\right\rangle,\right.
$$

which has causal support, will finally lead to the desired result eq. (7). For this purpose, the Fourier transform of $\Delta_{n}(x)$ has to be calculated. This can be done inductively. For $n=2$, we first evaluate the Fourier transform of $\Delta_{2}^{-}(x):=\Delta^{-}(x)^{2}$ given by the convolution

$$
\begin{aligned}
\hat{\Delta}_{2}^{-}(k) & =\int \frac{\mathrm{d}^{4} q}{(2 \pi)^{4}} \hat{\Delta}^{-}(q) \hat{\Delta}^{-}(k-q) \\
& =-\frac{1}{(2 \pi)^{2}} \int \mathrm{d}^{4} q \Theta\left(-q^{0}\right) \delta\left(q^{2}\right) \Theta\left(q^{0}-k^{0}\right) \delta\left((k-q)^{2}\right) .
\end{aligned}
$$

The integral vanishes outside the closed backward lightcone due to Lorentz invariance and the two $\Theta$-distributions in Eq. (25). Therefore one can go to a Lorentz frame where $k=\left(k^{0}<0, \overrightarrow{0}\right)$, and using the abbreviation $E=\sqrt{\vec{q}^{2}}=|\vec{q}|$ and exploiting the $\delta$-distribution in Eq. (25) one obtains

$$
\begin{aligned}
\hat{\Delta}_{2}^{-}\left(k^{0}<0, \overrightarrow{0}\right) & =-\frac{1}{(2 \pi)^{2}} \int \frac{\mathrm{d}^{3} q}{2 E} \delta\left(\left(k^{0}\right)^{2}+2 k^{0} E\right) \Theta\left(-E-k^{0}\right) \\
& =-\frac{1}{2(2 \pi) k^{0}} \int \mathrm{d}|\vec{q}||\vec{q}| \delta\left(\frac{k^{0}}{2}+|\vec{q}|\right) \Theta\left(-|\vec{q}|-k^{0}\right) \\
& =-\frac{1}{4(2 \pi)} \Theta\left(-k^{0}\right) \Theta\left(\left(k^{0}\right)^{2}\right),
\end{aligned}
$$

and for arbitrary $k$ one gets the intermediate result

$$
\hat{\Delta}_{2}^{-}(k)=-\frac{1}{8 \pi} \Theta\left(-k^{0}\right) \Theta\left(k^{2}\right) .
$$

According to Eq. (24) we have $\Delta_{2}(x)=\Delta^{-}(x)^{2}-\Delta^{+}(x)^{2}$, and from Eq. (11) follows $\Delta^{-}(-x)=-\Delta^{+}(x)$, therefore we obtain for the Fourier transform of $\Delta_{2}(x)$

$$
\hat{\Delta}_{2}(k)=\frac{1}{8 \pi} \operatorname{sgn}\left(k^{0}\right) \Theta\left(k^{2}\right) .
$$

It is obvious that $\hat{\Delta}_{2}(k)$ is related to the real part of Eq. (4).

\section{Point Splitting Method in Momentum Space}

Instead of introducing a mass term as regulator of the distribution $\hat{\Delta}_{2}(k)$, we present here another strategy, namely a point splitting procedure in momentum space, which also leads to the desired result. As was shown in Epstein and Glaser [5] and Dütsch et al. [25], the massless causal distribution $\hat{\Delta}_{2}(k)$ can be split in analogy to 
Eq. (18) by computing a retarded part $\hat{\Delta}_{2, \text { ret }}^{q}(k)$ in momentum space of $\Delta_{2}(x) \mathrm{e}^{i q x}$ in configuration space via the subtracted dispersion relation [26]

$$
\hat{\Delta}_{2, \text { ret }}^{q}(k)=\frac{i}{2 \pi} \int \mathrm{d} t \frac{\hat{\Delta}_{2}(t k+q)}{(t-i 0)(1-t+i 0)}
$$

for $k-q \in V^{+}, q^{2}<0$ and performing a limit

$$
\hat{\Delta}_{2}^{\text {ret }}(k)=\lim _{q \rightarrow 0}\left[\hat{\Delta}_{2, \text { ret }}^{q}(k)+c_{q}\right],
$$

where $c_{q}$ is a diverging $q$-dependent constant (i.e., $c_{q}$ is independent of $k$ ) which can be chosen in such a way that the limit in Eq. (30) exists. Note that $\hat{\Delta}_{2}(k+q)$ is the Fourier transform of $\Delta_{2}(x) \mathrm{e}^{i q x}$. Therefore, one has to compute $\left(k \in V^{+}\right)$

$$
\hat{\Delta}_{2}^{\text {ret }}(k)=\lim _{q \rightarrow 0}\left\{\frac{i}{16 \pi^{2}} \int \mathrm{d} t \frac{\operatorname{sgn}\left(t k^{0}+q^{0}\right) \Theta\left((t k+q)^{2}\right)}{(t-i 0)(1-t+i 0)}+c_{q}\right\} .
$$

The roots of $(t k+q)^{2}=t^{2} k^{2}+2 t(k q)+q^{2}=0$ are

$$
t_{1,2}=\frac{1}{k^{2}}(-k q \pm \sqrt{N})
$$

with $N=(k q)^{2}-k^{2} q^{2}>(k q)^{2}$ and $t_{1}>0, t_{2}<0$. The integral in (31) becomes

$$
\begin{aligned}
& \left\{\frac{-i}{16 \pi^{2}} \int_{-\infty}^{t_{2}} \mathrm{~d} t+\frac{i}{16 \pi^{2}} \int_{t_{1}}^{\infty} \mathrm{d} t\right\}\left\{\frac{1}{t}-P \frac{1}{t-1}-i \pi \delta(t-1)\right\} \\
= & \frac{i}{16 \pi^{2}}\left(-\log \left|t_{2}\right|+\log \left|t_{2}-1\right|-\log \left|t_{1}\right|+\log \left|t_{1}-1\right|-i \pi\right) .
\end{aligned}
$$

Since $t_{1,2} \rightarrow 0$ for $q \rightarrow 0$ the $\operatorname{logarithms} \log \left|t_{1,2}-1\right|$ vanish in this limit and the integral reduces to

$$
-\frac{i}{16 \pi^{2}}\left(\log \left|t_{1} t_{2}\right|+i \pi\right)=\frac{i}{16 \pi^{2}}\left(\log \frac{k^{2}}{\left|q^{2}\right|}-i \pi\right) .
$$

Adding $c_{q}=\frac{i}{16 \pi^{2}} \log \frac{\left|q^{2}\right|}{\mu_{\mathcal{R}}^{2}}$ to Eq. (34) leads after analytic continuation to the (covariant) splitting solution

$$
\hat{\Delta}_{2}^{r e t}(k)=\frac{i}{16 \pi^{2}} \log \left(-\frac{k^{2}+i k^{0} 0}{\mu_{\mathcal{R}}^{2}}\right),
$$

in accordance with the well-known result for $\Gamma_{2}^{\mathcal{R}}$ given by Eq. (4). 


\section{Results for Higher Loops}

We proceed with the Fourier transform of $\Delta^{-}(x)^{3}=: \Delta_{3}^{-}(x)$

$$
\begin{aligned}
\hat{\Delta}_{3}^{-}(k) & =\frac{i}{(2 \pi)^{3}} \int \mathrm{d}^{4} q \Theta\left(-q^{0}\right) \delta\left(q^{2}\right) \hat{\Delta}_{2}^{-}(k-q) \\
& =-\frac{i}{4(2 \pi)^{4}} \int \mathrm{d}^{4} q \Theta\left(-q^{0}\right) \delta\left(q^{2}\right) \Theta\left(q^{0}-k^{0}\right) \Theta\left((k-q)^{2}\right)
\end{aligned}
$$

For $k=\left(k^{0}<0, \overrightarrow{0}\right)$ we obtain

$$
\begin{aligned}
\hat{\Delta}_{3}^{-}(k) & =-\frac{i}{4(2 \pi)^{4}} \int \frac{\mathrm{d}^{3} q}{2 E} \Theta\left(-E-k^{0}\right) \Theta\left(\left(k^{0}\right)^{2}+2 k^{0} E\right)-\frac{i}{4(2 \pi)^{3}} \int_{0}^{-k^{0} / 2} \mathrm{~d}|\vec{q} \| \vec{q}| \\
& =-\frac{i}{32(2 \pi)^{3}} k^{2}
\end{aligned}
$$

hence

$$
\hat{\Delta}_{3}^{-}(k)=-\frac{i}{32(2 \pi)^{3}} k^{2} \Theta\left(-k^{0}\right) \Theta\left(k^{2}\right) .
$$

Inserting a factor $(k-q)^{2}=\left(k^{0}\right)^{2}+2 k^{0} E$ in Eq. (37), corresponding to a replacement $\hat{\Delta}_{n}^{-}(k) \rightarrow \hat{\Delta}_{n+1}^{-}(k)$ in Eq. (36), leads after a short inductive calculation to $(n \geqslant 2)$

$$
\hat{\Delta}_{n}^{-}(k)=\frac{i^{n}\left(k^{2}\right)^{n-2}}{4^{n-1}(n-1) !(n-2) !(2 \pi)^{2 n-3}} \Theta\left(-k^{0}\right) \Theta\left(k^{2}\right)
$$

or

$$
\hat{\Delta}_{n}(k)=\frac{\left(k^{2}\right)^{n-2}}{4^{n-1}(n-1) !(n-2) !(2 \pi)^{2 n-3}} \operatorname{sgn}\left(k^{0}\right) \Theta\left(k^{2}\right) .
$$

Keeping in mind that $\left(k^{2}\right)^{n-2}$ in Fourier space corresponds to the local operator $(-\square)^{n-2}$ in configuration space, a retarded part of $\Delta_{n}$ can be obtained directly from the retarded solution $\Delta_{2}^{\text {ret }}$ calculated above for $\Delta_{2}$ in momentum space, since $\left(k^{2}\right)^{n-2} \hat{\Delta}_{2}^{\text {ret }}(k)$ is a retarded part of $\left(k^{2}\right)^{n-2} \hat{\Delta}_{2}(k)$. The actual calculation is straightforward and leads after analytic continuation of $\hat{\Delta}_{2}^{\text {ret }}(k)$ to the desired result

$$
\Gamma_{n}^{\mathcal{R}}(k)=-\frac{(-i)^{n+1}\left(k^{2}\right)^{n-2}}{4^{n-1}(2 \pi)^{2 n-2}(n-1) !(n-2) !} \log \left(-\frac{k^{2}+i 0}{\mu_{\mathcal{R}}^{2}}\right)+\sum_{i=0}^{n-2} c_{i}^{\mathcal{R}}\left(k^{2}\right)^{i}
$$

for $n \geqslant 2$.

\section{Acknowledgements}

The author wishes to thank Cyrill von Arx for generating the figure. This work was supported by the Swiss National Science Foundation. 


\section{References}

1. Pauli, W., Villars, F.: On the invariant regularization in relativistic quantum theory. Rev. Mod. Phys. 21, 434 (1949)

2. 't Hooft, G., Veltman, B.: Regularization and renormalization of gauge fields. Nucl. Phys. B44, 189 (1972)

3. Schwinger, J.: On gauge invariance and vacuum polarization. Phys. Rev. 82, 664 (1951)

4. Scharf, G.: Finite Quantum Electrodynamics. 2nd edn, Springer, Berlin Heidelberg New York (1995)

5. Epstein, H., Glaser, V.: The role of locality in perturbation theory. Ann. Poincaré Phys. Theor. A19, 211 (1973)

6. Aste, A.: The two loop master diagram in the causal approach. Ann. Phys. 257, 158 (1997)

7. Cheng, T.-P., Li, L.-F.: Gauge Theory of Elementary Particle Physics. Oxford University Press, New York (1984)

8. Källén, G., Sabry, A.: Fourth order vacuum polarization. Dan. Mat. Fys. Medd. 29(17), 1 (1955)

9. Tkachov, F.V.: A theorem on analytical calculability of four loop renormalization group functions. Phys. Lett. B100, 65 (1981)

10. Chetyrkin, K.G., Tkachov, F.V.: Integration by parts: The algorithm to calculate beta functions in 4 loops. Nucl. Phys. B192, 159 (1981)

11. Bauberger, S., Berends, F.A., Böhm, M., Buza, M.: Analytical and numerical methods for massive two loop selfenergy diagrams. Nucl. Phys. B434, 383 (1995)

12. Berends, F.A., Davydychev, A.I., Ussyukina, N.I.: Threshold and pseudothreshold values of the sunset diagram. Phys. Lett. B426, 95 (1998)

13. Fleischer, J., Kotikov, A.V., Veretin, O.L.: Analytic two loop results for selfenergy type and vertex type diagrams with one nonzero mass. Nucl. Phys. B547, 343 (1999)

14. Post, P., Tausk, J.B.: The sunset diagram in SU(3) chiral perturbation theory. Mod. Phys. Lett. A11, 2115 (1996)

15. Davydychev, A.I., Smirnov, V.A.: Threshold expansion of the sunset diagram. Nucl. Phys. B554, 391 (1999)

16. Caffo, M., Czyż, H., Remiddi, E.: The threshold expansion of the two loop sunrise selfmass master amplitudes. Nucl. Phys. B611, 503 (2001)

17. Passarino, G.: An approach toward the numerical evaluation of multiloop Feynman diagrams. Nucl. Phys. B619, 257 (2001)

18. Aste, A., Trautmann, D.: Finite calculation of divergent selfenergy diagrams. Can. J. Phys. 81, 1433 (2003)

19. Laporta, S., Remiddi, E.: The analytic value of a 4-loop sunrise graph in a particular kinematical configuration. Nucl. Phys. B704, 349 (2005)

20. Laporta, S.: High-precision calculation of multiloop Feynman integrals by difference equations. Int. J. Mod. Phys. A15, 5087 (2000)

21. Groote, S., Pivovarov, A.A.: Threshold expansion of Feynman diagrams within a configuration space technique. Nucl. Phys. B580, 459 (2000)

22. Groote, S., Körner, J.G., Pivovarov, A.A: On the evaluation of a certain class of Feynman diagrams in $x$-space: Sunrise-type topologies at any loop order. hep-ph/0506286

23. Streater, R.F., Wightman, A.S.: PCT, Spin, Statistics and All That. Benjamin-Cummings Publishing Company, Reading (1964)

24. Constantinescu, F.: Distributions and Their Applications in Physics. Pergamon Press, New York (1980) 
25. Dütsch, M., Hurth, T., Krahe, F., Scharf, G.: Causal construction of Yang-Mills theories: II. Nuovo Cim. A107, 375 (1994)

26. Aste, A., G. Scharf, G., Walther, U.: Power counting degree versus singular order in the Schwinger model. Nuovo Cim. A111, 323 (1998) 